\title{
О НЕКОТОРЫХ АСПЕКТАХ МЕТОДОВ ГЕНЕРИРОВАНИЯ ТОЧНЫХ АКСИАЛЬНО-СИММЕТРИЧНЫХ СТАЦИОНАРНЫХ РЕШЕНИЙ УРАВНЕНИЙ ЭЙНШТЙНА
}

\author{
(Представил П. Кард)
}

\section{Введение}

Пусть $\left(V_{4}, g\right)$ допускает абелеву группу изометрий $G_{2}$ и описывает аксиально-симметричное (AC) стационарное вакуумное гравитационное поле. Қак обычно принято, рассмотрим это поле в координатных системах, присоединенных к соответствующим киллинговым векторным полям $\xi^{\mu}$ и $\xi^{v}$, причем координаты $x^{A}\left(A, B, \ldots=3,4 ; x^{4}=c x^{0}\right)$ выде(0) (3)

лены так, чтобы градиенты их координатных поверхностей были ортогональны градиентам поверхностей $x^{M}=$ const, определяемых траекториями группы $G_{2}(M, N, \ldots=1,2)$. Получаемую стандартную метрическую форму зададим в виде

где

$$
d s^{2}=\lambda^{-1}\left[\mathrm{e}^{2(\mu+v)} d \mathrm{~s}^{2}+W^{2}\left(d x^{3}\right)^{2}\right]-\lambda\left[A d x^{3}+c d x^{0}\right]^{2},
$$

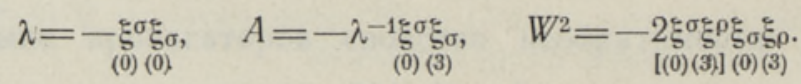

Инварианты (2), а также величины $\mu$ и $v$ являются теперь функциями только координат $x^{M}$ и, таким образом, проблема решения уравнений Эйнштейна $R_{\alpha \beta}=0$ сводится к двумерной задаче, которая может быть сформулирована в евклидовом $V_{2}$, где можно пользоваться либо декартовыми координатами $x^{M}$, либо комплексными координатами $y^{1}=\frac{1}{\sqrt{2}}\left(x^{1}+i x^{2}\right), y^{2}=y^{1 *}, \quad$ так что для $V_{2}$ метрика имеет вид

$$
d \mathrm{~s}^{2}=\left(d x^{1}\right)^{2}+\left(d x^{2}\right)^{2}=2 d y^{1} d y^{2} .
$$

Уравнения поля распадаются на две группы: $R_{A B}=0$ определяют величины $\lambda, A$ и,$W$, а затем остальные уравнения дают функции $\mu$ и $v$, выраженные через эти инварианты. Тройке действительных функций $(\lambda, A, W)$ равноценна также пара $(\varepsilon, W)$, где

$$
\varepsilon=\lambda+i \Psi
$$

- комплексный потенциал Эрнста, а твист-потенциал $\Psi$ определяется из соотношений

$$
i W \partial_{1} \Psi=\lambda^{2} \partial_{1} A, \quad-i W \partial_{2} \Psi=\lambda^{2} \partial_{2} A
$$


(здесь и в дальнейшем операторы $\partial_{1}, \partial_{2}=\partial_{1}{ }^{*}$ означают дифференцирование по комплексным координатам $y^{1}$ и $y^{2}$, звездочка означает комплексное сопряжение).

Уравнениям $R_{A B}=0$ можно придать разные виды. Но в любом случае из них выделяется уравнение

$$
\partial_{1} \partial_{2} W=0
$$

после чего в общем случае остаются еще по существу два нелинейных дифференциальных уравнения второго порядка для двух независимых действителыных функций, зависящих от двух переменных. Для потенциала \& имеем одно комплексное уравнение

$$
\left(\varepsilon+\varepsilon^{*}\right)\left[\partial_{1}\left(W \partial_{2} \varepsilon\right)+\partial_{2}\left(W \partial_{1} \varepsilon\right)\right]=4 W \partial_{1} \varepsilon \partial_{2} \varepsilon
$$

Огметим еще, что, как известно, именно уравнение (6) делает возможным рассмотрение $\mathrm{AC}$ стационарных вакуумных полей также в определенном евклидовом $V_{3}$ с метрической формой

$$
d l^{2}=\mathrm{e}^{2 v} d \mathrm{~s}^{2}+W^{2}\left(d x^{3}\right)^{2} .
$$

Функция $v$ определяется в силу евклидовости $V_{3}$ только функцией $W$, в то время как функция $\mu$ в (1) является характеристикой искривленности физического 3-пространства и определяется всей совокупностью величин $(\lambda, A, W)$.

В последнее время развиты разные новые подходы к генерированию новых точных решений уравнения (7) или равноценных ему систем нелинейных уравнений. Здесь могут быть применены, например, методы обратной задачи рассеяния [ $\left.{ }^{1}\right]$ и преобразований Бэклунда $\left[{ }^{2,3}\right]$. При любом методе генерирования решений мы имеем по существу алгоритм, определяющий переход $(\lambda, A, W) \rightarrow\left(\lambda^{\prime}, A^{\prime}, W^{\prime}\right)$ или $(\mathcal{E}, W) \rightarrow$ $\rightarrow\left(\mathcal{E}^{\prime}, W^{\prime}\right)$. В данной статье мы поставили себе задачу проанализировать некоторые аспекты определенного типа методов генерирования новых решений, характеризуемого в обобщенном виде алгоритмом вида

$$
\begin{aligned}
& \mathcal{E}^{\prime}=F \mathcal{E}, \\
& W^{\prime}=H W,
\end{aligned}
$$

где $F$ и $H$ - комплексная и действительная функции соответственно, определяемые конкретным методом генерирования.

\section{Некоторые общие теоремы}

Выясним прежде всего некоторые основные свойства алгоритмов типа (9) - (10) при $H \neq$ const.

Поскольку в силу (6) $W$ и $W^{\prime}$ должны быть гармоническими функциями в 2-пространстве $V_{2}$, имеем сразу общее условие

$$
H=\left(W_{(1)}^{\prime}+W_{(2)}^{\prime}\right)\left(W_{(1)}+W_{(2)}\right)^{-1},
$$

где $W_{(1)}^{\prime}$ и $W_{(1)}$ - произвольные функции координаты $y^{1}$, а $W_{(2)}^{\prime}=W_{(1)}^{\prime *}, W_{(2)}=W_{(1)}^{*}$. Более конкретный вид функции $H$ получается при определенных условиях, налагаемых на $H$ применяемым методом.

Пусть

$$
H=H_{(1)} H_{(2)},
$$


где $H_{(1)}$ - функция только координаты $y^{1}$, а $H_{(2)}=H_{(1)}^{*}$. Так как в AC стационарном случае при $W \neq$ const всегда $\quad W_{(2)}=W_{(1)}^{*} \neq$ const, то без ограничения общности из (11) и (12) следует

$$
H_{(1)}=\left(1+i h \sqrt{2} W_{(1)}\right)^{-1}=H_{(2)}^{*}
$$

и

$$
W_{(1)}^{\prime}=H_{(1)} W_{(1)}=W_{(2)}^{\prime *},
$$

где $h$ - действительная постоянная.

Интересно отметить, что так как в силу (12) всегда $\partial_{1} \partial_{2} \ln H=0$, то в рассматриваемом случае можно определить новую («дуальную») функцию $\gamma$ так, чтобы имело место

$$
\partial_{1} \ln H=\partial_{1} \ln \gamma, \quad \partial_{2} \ln H=-\partial_{2} \ln \gamma .
$$

Интегрируя эти соотношения, получаем

$$
\gamma=H_{(1)} H_{(2)}^{-1}=\left(\gamma^{*}\right)^{-1}
$$

Далее прямые вычисления дают соотношения

$$
\partial_{1}\left(\ln W^{\prime}\right)=\gamma \partial_{1}(\ln W), \quad \partial_{2}\left(\ln W^{\prime}\right)=\gamma^{-1} \partial_{2}(\ln W),
$$

а отсюда в свою очередь вытекают совместимые дифференциальные уравнения для $\gamma$ :

$$
\partial_{1} \gamma=\gamma(\gamma-1) \partial_{1}(\ln W), \quad \partial_{2} \gamma=\left(\partial_{1} \gamma^{-1}\right)^{*} .
$$

Наоборот, из этих уравнений для функции $\gamma$ вытекает соотношение (10) при условии (12).

Таким образом, доказана

Т еорем а 1. При дополнительном условии (12) для Н нахождение с помощью алгоритма (10) новой функции $W^{\prime}$ эквивалентно нахождению функций $W$ и ү из дифференциальных уравнений (17)-(18), причем ү и Н связаны соотношением (16).

Если в (13)-(14) $h \neq 0$, то характер пространственных координат в евклидовом $V_{3}$ с метрикой (8) изменяется, что нужно учитывать при анализе получаемых новых решений. Ниже изложим один из способов изучения такого изменения пространственных координат.

Пусть в $V_{3}$ введены как для исходного, так и для тенерируемого решения вейлевы канонические координаты $x^{1}=\varrho, x^{2}=z$ и $x^{1^{\prime}}=Q^{\prime}$, $x^{2}=z^{\prime}$, т. е. пусть имеем $\left(y=y^{1}, y^{\prime}=y^{1^{\prime}}\right)$

$$
W=\frac{1}{\sqrt{2}}\left(y+y^{*}\right)=\varrho, \quad W^{\prime}=\frac{1}{\sqrt{2}}\left(y^{\prime}+y^{\prime *}\right)=\varrho^{\prime} .
$$

В силу (19) преобразование (14) получает вид

$$
y^{\prime}=y(1+i h y)^{-1}=-i K y(\sqrt{2 y}-i K)^{-1},
$$

где $K=\sqrt{2} h^{-1}$. . Для функции $\gamma(16)$ получается

$$
\gamma=-[\varrho-i(z-K)][\varrho+i(z+K)]^{-1} \text {. }
$$

Прямыми выкладками доказывается теперь

Т еорема 2. Любое преобразование типа

можно представить 
как последовательность т. н. КІ ции, причем $K_{\mathrm{I}}$-трансляция определяется формулами

$$
\bar{y}=y-i h^{-1}=\frac{i}{\sqrt{2}} \bar{R} \mathrm{e}^{-i \bar{\theta}} \quad(\varrho=\bar{R} \sin \bar{\theta}, z=\bar{R} \cos \bar{\theta}+K),
$$

К-инверсия -

$$
y^{\prime \prime}=\left(h^{2} \bar{y}\right)^{-1} \quad\left(\bar{R} R^{\prime \prime}=K^{2}, \bar{\theta}+\theta^{\prime \prime}=\pi\right),
$$

а К КІ-трансляция -

$$
y^{\prime}=y^{\prime \prime}-i h^{-1} \quad\left(Q^{\prime}=R^{\prime \prime} \sin \theta^{\prime \prime}, z^{\prime}=R^{\prime \prime} \cos \theta^{\prime \prime}-K\right) .
$$

Из теоремы 2 получается, во-первых, наглядное представление о характере преобразования (20) вейлевых координат в $V_{3}$. Во-вторых, на основе теоремы 2 можно получить ряд полезных формул, позволяющих упростить вычисления. Именно, в силу (22) мы имеем возможность перехода от вейлевых координат $y^{1}=y=\frac{1}{\sqrt{2}}(\varrho+i z) \quad$ к специальным сферическим координатам $\bar{R}$ и $\bar{\theta}$ в $V_{3}$. Тогда вводятся новые операторы дифференцирования по формуле

$$
\partial_{1}=\frac{1}{\sqrt{2}} \mathrm{e}^{i \bar{\theta}}\left(\bar{R}^{-1} \frac{\partial}{\partial \bar{\theta}}-i \frac{\partial}{\partial \bar{R}}\right),
$$

а для функций $H$ и $\gamma$ получаются формулы

$$
H=K^{2}(\bar{R})^{-2}, \quad \gamma=\mathrm{e}^{2 i \bar{\theta}} .
$$

\section{О методе Нейгебауэра $(H=1)$}

Одним конкретным видом алгоритма типа (9) является метод генерирования новых решений, данный Нейгебауэром $\left[{ }^{4,5}\right]$. Хотя в этом методе имеет место $W^{\prime}=W$, т. е. $H=1$, но комплексная функция $F$ является по существу функционалом, в который входят пары «генерирующих потенциалов» $\left(\gamma_{k}, x_{k}\right)(k=0,1, \ldots, 2 N ; N=1,2, \ldots)$, причем функции $\gamma_{k}$ определяются именно решениями уравнений (18) с разными постоянными интегрирования, т. е. эти функции имеют вид (16) с разными $h_{k}$ (или $K_{k}$ ). При этом $\gamma_{0}=1$, т. е. $h_{0}=0$. Каждому конкретному $\gamma_{k}$ соответствует определенная величина ${ }^{*} x_{k}=\left(x_{k}{ }^{*}\right)^{-i}$, которая является решением уравнений

$$
\begin{gathered}
\partial_{1} \varkappa_{k}=\left(\mathcal{E}+\mathcal{E}^{*}\right)^{-1}\left[\gamma_{k}^{1 / 2}\left(x_{k}^{2} \partial_{1} \mathcal{E}-\partial_{1} \mathcal{E}^{*}\right)-\chi_{k} \partial_{1}\left(\mathcal{E}-\mathcal{E}^{*}\right)\right], \\
\partial_{2} \varkappa_{k}=\left[\partial_{1} x_{k}^{-1}\right]^{*},
\end{gathered}
$$

т. е. определяется исходным решением. После интегрирования уравнений (27), условиями интегрируемости которых являются уравнения поля, т. е. (7), задача вычисления нового решения становится чисто алгебраической.

Если $\gamma_{k}=1$, то уравнения (27) легко интегрируются. Но в общем случае $\gamma_{k} \neq 1$ интегрирование этих уравнений наталкивается на труд-

* В $\left[{ }^{2}\right]$ имеет место $\alpha \gamma^{-1 / 2} \equiv \chi$, а в $\left[{ }^{5}\right]-\alpha \equiv \chi$. 
ности, обусловленные как видом исходного решения $\mathcal{E}$, так и неудобным видом функции $\gamma_{k}$ в координатах $\left(y^{1}, y^{2}\right)$, даже если $y^{1}=y$ соответствует вейлевым каноническим координатам (см. формулы (16), (13) и (19)). Для приведения уравнений (27) к более удобному виду предложим перейти от вейлевых координат к сферическим $(\bar{R}, \bar{\theta})$ по формулам (22). Продемонстрируем этот метод в случае, если исходные решения являются статическими, т. е. при условии

$$
\mathcal{E}=\mathcal{E}^{*}=\mathrm{e}^{2 U}
$$

В силу (28) уравнения (27) интегрируются, причем всегда можно задать функции $\varkappa_{k}$ в виде

$$
x_{k} \equiv \mathrm{e}^{i \omega_{k}}=\left(-i p_{k} \mathrm{e}^{2 \Phi_{k}}+q_{k}\right)\left(i p_{k} \mathrm{e}^{2 \Phi_{k}}+q_{k}\right)^{-1},
$$

где, в отличие от вида этой формулы в [ $\left.{ }^{5}\right]$, мы ввели две действительные постоянные $p_{k}$ и $q_{k}$. Формула (29) охватывает и частные случаи $\varkappa_{k}=+1\left(p_{k}=0, q_{k} \neq 0\right)$ и $x_{k}=-1\left(q_{k}=0, p_{k} \neq 0\right)$, когда $U$ и $\gamma_{k}$ произвольны. Для $\Phi_{k}$ имеем уравнения [ $\left.{ }^{5}\right]$

$$
\partial_{1} \Phi_{k}=\gamma_{k}^{1 / 2} \partial_{1} U, \quad \partial_{2} \Phi_{k}=\gamma_{k}^{1 / 2} \partial_{2} U
$$

Отсюда видим, что при $\gamma_{k}=1$ функция $\Phi_{k}$ в формуле (29) попросту заменяется на $U$.

Введем теперь при $\gamma_{k} \neq 1$ для вычисления каждого $x_{k}$ по формулам (22) свои специальные сферические координаты $\left(R_{k}, \theta_{k}\right)$ :

$$
R_{k} \mathrm{e}^{-i \theta_{k}}=R \mathrm{e}^{-i \theta}-K_{k}
$$

Для вейлевых координат имеем теперь $\varrho=R \sin \theta, z=R \cos \theta$, а $K_{k}$ входит в выражение (21) соответствующей величины $\gamma_{k}$. В силу (26) имеем

$$
\gamma_{k}=\mathrm{e}^{2 i \theta_{k}}
$$

а учитывая еще вид дифференциального оператора $\partial_{1}$ (25) получаем уравнения (30) в виде

$$
\begin{gathered}
\frac{\partial \Phi_{k}}{\partial R_{k}}=\cos \theta_{k} \frac{\partial U}{\partial R_{k}}-R_{k}^{-1} \sin \theta_{k} \frac{\partial U}{\partial \theta_{k}} \\
\frac{\partial \Phi_{k}}{\partial \theta_{k}}=R_{k} \sin \theta_{k} \frac{\partial U}{\partial R_{k}} f \cos \theta_{k} \frac{\partial U}{\partial \theta_{k}}
\end{gathered}
$$

Как известно, в АС статическом вакуумном случае уравнение (7) переходит в уравнение Лапласа для $U$ в координатах 3-пространства $V_{3}$. Пусть такая определенная гармоническая функция теперь задана в сферических координатах $(R, \theta)$ в $V_{3}$. Пользуясь преобразованиями координат (31), всегда можно выразить функцию $U$ также в координатах $\left(R_{k}, \theta_{k}\right)$. Подставляя такую $U$ в уравнения (33) и решая эти уравнения, получим $\Phi_{k}=\Phi_{k}\left(R_{k}, \theta_{k}\right)$. Совершая обратный переход к координатам $(R, \theta)$, получим $\Phi_{k}=\Phi_{k}(R, \theta)$, а тогда в силу $(29)$ имеем и $x_{k}=$ $=x_{k}(R, \theta)$.

Для практических вычислений полезно вывести нз формулы (31) соотношения

$$
R_{k}=R Y_{k}^{-1}, \quad \sin \theta_{k}=Y_{k} \sin \theta, \quad \cos \theta_{k}=Y_{k}\left(\cos \theta-t_{k}\right),
$$




$$
R=R_{k}\left(Y_{k}^{\prime}\right)^{-1}, \quad \sin \theta=Y_{k}^{\prime} \sin \theta_{k}, \quad \cos \theta=Y_{k}^{\prime}\left(\cos \theta_{k}+t_{k}^{\prime}\right),
$$

где для $Y_{k}$ и $t_{k}$ имеем

$$
\begin{gathered}
Y_{k}=\left(1-2 t_{k} x+t_{k}^{2}\right)^{-1 / 2}=\sum_{n=0} P_{n}(x) t_{k}^{n}, \\
t_{k}=K_{k} R^{-1}, \quad x=\cos \theta,
\end{gathered}
$$

$P_{n}(x)$ - полиномы Лежандра. Величины со штрихом имеют мѐсто для обратного перехода, причем для $Y^{\prime}{ }_{k}$ и $t^{\prime}{ }_{k}$ получаем такие же формулы, как для $Y_{k}$ и $t_{k}$, только переменные $(R, \theta)$ заменены на $\left(R_{k}, \theta_{k}\right)$ и величина $t_{k}$ на $t_{k}^{\prime}=K_{k} R_{k}{ }^{-1}$.

Из конкретных выражений для $U$ представляют наибольший интеpec, естественно, те, которые являются потенциалами поля пространственно компактного («островного типа») источника. Тогда функцию $U$ можно задать в виде мультипольного разложения либо в координатах $(R, \theta)$, либо в координатах $\left(R_{k}, \theta_{k}\right)$ :

$$
U=\sum_{s=0} a_{s} R^{(s+1)} P_{s}(\cos \theta)=\sum_{p=0} a_{p}^{(k)} R_{k}^{-(p+1)} P_{p}\left(\cos \theta_{k}\right)
$$

причем мультипольные моменты $a_{s}$ и $a_{p}{ }^{(k)}$ связаны соотношениями

$$
a_{s}=\sum_{p=0}^{s} C_{s}^{p}\left(K_{k}\right)^{s-p} a_{p}^{(k)}
$$

где $C_{s}{ }^{p}$ - биномиальные коэффициенты. Подставляя выражение (38) в уравнения (33), после интегрирования получим

$$
\Phi_{k}=\sum_{p=0} a_{p}^{(k)} R_{k}^{-(p+1)} P_{p+1}\left(\cos \theta_{k}\right)
$$

откуда после обратного перехода в координаты $(R, \theta)$ имеем

$$
\Phi_{k}=Y_{k}^{-1} \sum_{s=0} \bar{a}_{s}^{(k)} R^{-(s+1)} P_{s+1}(\cos \theta), \quad \bar{a}_{s}^{(k)}=\sum_{p=0}^{s}\left(K_{k}\right)^{s-p} a_{p} .
$$

Этим завершается нахождение функции $\Phi_{k}$, а в силу (29) тем самым и функции $x_{k}$ по заданному мультипольному разложению.

Определенную ценность (см. ниже) могут иметь и потенциалы $U$ для «некомпактных» источников, например,

$$
U=-a \ln \varrho=-a \ln (R \sin \theta)
$$

(потенциал поля «бесконечной нити») или

$$
U=-a z=-a R \cos \theta
$$

(потенциал поля «бесконечной и бесконечно тонкой плоскости»). Подставляя выражения (42) и (43) в уравнения (33), после интегрирования с учетом (35) получим соответственно

$$
\begin{gathered}
\Phi_{k}=-a \ln \tan \frac{\theta_{k}}{2}= \\
=-\frac{a}{2} \ln \left\{\left[1-\left(\cos \theta-t_{k}\right) Y_{k}\right]\left[1+\left(\cos \theta-t_{k}\right) Y_{k}\right]^{-1}\right\}
\end{gathered}
$$

и

$$
\Phi_{k}=a R_{k}=a R Y_{k}^{-1}
$$




\section{О преобразованиях Бэклунда в случае $H \neq$ const}

B $\left[{ }^{2}\right]$ определена операция $I$, генерирующая из заданных AC стационарных вакуумных решений новые и имеющая вид (в наших обозначениях)

$$
\left(\lambda^{\prime}\right)^{-1} \partial_{1} \mathcal{E}^{\prime}=x \gamma^{1 / 2} \lambda^{-1} \partial_{1} \varepsilon, \quad\left(\lambda^{\prime}\right)^{-1} \partial_{1} \mathcal{E}^{\prime *}=x^{-1} \gamma^{1 / 2} \lambda^{-1} \partial_{1} \mathcal{E}^{*},
$$

причем, в общем случае $(\gamma \neq 1)$ сюда добавляются и уравнения (17), определяющие изменение $W \rightarrow W^{\prime}$, т. е. функцию $H \neq$ const. B cooтношениях (46) величины $x=\chi_{k}$ и $\gamma=\gamma_{k}$ имеют тот же смысл, что и выше, т. е. являются решениями уравнений (27) и (18). Вместе с операцией $S$, относительно которой уравнение (7) инвариантно и которая состоит в замене величин $\varepsilon_{+}=\lambda^{-1} W-A$ и $\varepsilon_{-}=\lambda^{-1} W+A$ на $\varepsilon$ и $\varepsilon^{*}$ и наоборот, операция $I$ позволяет конструировать определенные «преобразования Бэклунда», дающие возможность генерировать из определенного исходного решения целую серию новых **. Метод Нейгебауэра генерирования новых решений типа (9) и (10) при $H=1$, рассмотренный нами выше, по существу содержит операции $I$ и $S$ как составные элементы $\left[{ }^{4,5}\right]$.

В случае исходного статического решения (28). интегрирование уравнения (46) при $x^{2} \neq 1$ дает

$$
\varepsilon^{\prime}=i(L \varkappa+M),
$$

где $L, M$ - действительные постоянные, а $\approx$ имеет вид $(29)$, т. е. задается при $\gamma \neq 1$ через функцию $\Phi$, а при $\gamma=1$ по той же формуле через $U$. Подчеркнем, что поскольку в случае получаемого потенциала Эрнста $\mathcal{E}^{\prime}=\lambda^{\prime}+i \Psi^{\prime}$ функции $\lambda^{\prime}$ и $\Psi^{\prime}$ функционально зависимы, то решение относится всегда к классу Элерса-Папапетру. При $x= \pm 1$ новое решение, генерируемое уравнениями (46) из статического, может быть лишь статическое, причем при $\gamma \neq 1$ новая функция $\pm U^{\prime}$ определяется из $U$ теми же формулами, что $\Phi$ из $U$, т. е. уравнениями (33), а при $\gamma=1$ получается $U^{\prime}= \pm U$.

Надо иметь в виду, что, рассматривая операцию (46) как однократный акт генерирования нового решения, в случае $\gamma \neq 1$ имеет место и переход к новой функции $W^{\prime}$, чему соответствует преобразование координат по формулам $(22)-(24)$. Поэтому, вычислив функцию Ф (или $\pm U^{\prime}$ при $x= \pm 1$ ) по уравнениям (33), нужно далее совершить и операцию K-инверсии по формуле $(23)$, т. е. перейти к координатам $\left(R_{k}^{\prime \prime}, \theta_{k}^{\prime \prime}\right)$. Можно показать, что если $U$ является гармоническим в координатах $\left(R_{k}, \theta_{k}\right)$, то $Ф-$ гармоническая функция в $\left(R_{k}^{\prime \prime}, \theta_{k}{ }^{\prime \prime}\right)$. Поэтому можно заключить, что не существует принципиальной разницы между случаями $\gamma \neq 1$ и $\gamma=1$ при генерировании новых решений с помощью операции (46) из статических.

В качестве простого наглядного примера $K$-инверсии может служить результат, получаемый из (45). Видим, что переход от (43) к (45) плюс $K$-инверсия переводят потенциал бесконечной плоскости в потенциал точечной массы. В рамках нашего анализа важно теперь иметь в виду, что такого рода «метаморфозы» потенциалов могут фактически быть составными элементами более сложных операций генери-

\footnotetext{
** В связи с этим отметим, что вычисление определенных вспомогательных функций, совпадающих с $\gamma$ и $x$, является составным элементом метода применення преобразований Бэклунда, развитого в $\left[^{3,6}\right]$. Как стало автору известно после завершения дан ной работы, в [ $\left.{ }^{6}\right]$ прнменялся, хотя в рамках совершенно другой постановки задачи, переход к новым переменным, в результате чего для величины, тождественной $\gamma$, фактически был получен также вид (32).
} 
рова́ния новых точных решений уравнений Әйнштейна. Например, поскольку в случае исходного статического решения $S$-операция имеет вид

$$
U^{\prime}=-U+\frac{1}{2} \ln W
$$

то в случае плоского исходного решения $(U=0) S$-операцияя даст по существу такое статическое решение, которое соответствует потенциалу (42) при $a=-1 / 2$. Если затем совершить операцию $I$, то получается уже переход к потенциалу типа (44), который в силу (29) и (47) определяет решение типа Элерса-Папапетру. Снова применив операцию $S$, получим решение класса Льюиса-Стокума, но с комплексными метрическими коэффициентами. Как показано в [ $\left.{ }^{7}\right]$, определенное преобразование может привести именно от решения такого типа к решению Kерра, а.тот же результат дает и сложное преобразование типа I'SIS в случае исходного плоского решения в $\left[{ }^{8}\right]$. Впоследствии выяснилось, что упомянутое сложное I'SIS эквивалентно применению алгоритма типа (9)-(10) при $H=1$, рассмотренного выше, причем предполагалось $N=1$ и исходная метрика принималась плоской $\left[{ }^{4,5}\right]$. Теперь, выделяя составные элементы, мы попыта́лись в некоторой мере раскрыть сущность получения метрики Керра из плоской метрики.

Приведенные выше соображения также конкретно демонстрируют, что конечное («актуальное») решение, представляющее физический интерес, может быть получено как результат применения целой цепи отдельных операций, причем промежуточные («виртуальные») решения могут явно быть «нефизическими». (Например, решение (48) при $U=0$ также трудно толковать физически, так как при заданном $W=\varrho$ оно не 'имеет нерелятивистского предела. Определенный предел в виде однородного поля получается лишь после перехода в другую координатную систему в $V_{3}$, причем в нерелятивистском пределе получаются в предельном пространстве $\stackrel{0}{V}_{3}$ декартовы координаты, а кривизна пространства-времени нулевая.)

Итак, возникает и задача физического анализа развитых в последнее время разных методов генерирования новых точных решений уравнений Эйнштейна. На наш взгляд, одним из возможных подходов к этой задаче является метод построения нерелятивистских аналогов изучаемых релятивистских решений $\left[{ }^{9,10}\right]$.

\section{ЛИТЕ РАТ У РА}

1. Белин ский В. А., 3 ах а ров В. Е., Ж. эксперим. и теор. физ., 77, вып. 1, $3-19$ (1979).

2. Neugebauer, G., J. Phys. A : Math. Gen., 12, № 4, L67-L70 (1979).

3. Harriso n, B. K., Phys. Rev. Lett., 41, № 18, 1197-1200 (1978).

4. Neu g e b a ue r, G., J. Phys. A : Math. Gen., 13, № 5, 1737-1740 (1980).

5. Neug e b a ue r, G., J. Phys. A : Math. Gen., 13, № 2, L19-L21 (1980).

6. H a r r is o n, B. K., Phys. Rev. D, 21, № 6, 1695-1697 (1980).

7. He r 1t, E., Gen. Relat. and Gravit., 9, № 8, $711-719$ (1978).

8. Neugebauer, G., Kramer, D., Exp. Techn. Phys., 28, № 1, 3-8 (1980).

9. К п пель А., В кн.: Современные проблемы общей теории относительности, Минск, АН БССР, 1979, с. 48-52.

10. Koppel, A., In: Abstracts of Contributed Papers for the Discussion Groups. 9th International Conference on General Relativity and Gravitation, July 14-19, 1980, FSU, Jena, GDR, 1980 , 1, p. $40-41$. 


\section{A. KOPPEL}

\section{EINSTEINI VÕRRANDITE TÄPSETE AKSIAALSUMMEETRILISTE STATSIONAAR- SETE LAHENDITE GENEREERIMISE MEETODITE MONINGATEST ASPEKTIDEST}

On analüüsitud mõningaid Einsteini võrrandite uute vaakuumlahendite leidmise meetodeid, mis on üldistatud kujul esitatavad ühise algoritmina $(9)-(10)$. On tõestatud kaks teoreemi juhu jaoks, kus muutujad funktsioonis $H$ on eraldatavad. Esimesest teoreemist järeldub, et vaadeldava algoritmi see erijuht on ekvivalentne "genereeriva potentsiaali» $Y(\gamma \neq$ const $)$ konstrueerimisega töödes $\left[{ }^{2,5}\right]$. Teisest teoreemist on tuletatud mõned oluriva potentsiaali» $x \equiv \alpha$ arvutamiseks on saadud artiklis $\left[{ }^{5}\right]$ arendatud meetodi «generee. arendatud Bäckl» $x \equiv \alpha$ arvutamiseks juhul, kui lähtelahend on staatiline. Artiklis [ $\left.{ }^{2}\right]$ ja $S$ - on uuritud staatilise ja kõverodi koostiselemente - operatsioone $I$ (kui $\gamma \neq$ const) analüüs lu analüüs lubab mõningal määral sügavamait mõista tasaselt meetrikalt Kerri meetrikale viivate Bäcklundi teisenduste olemust.

\section{A. KOPPEL}

\section{ON SOME ASPECTS OF THE GENERATION METHODS OF EXACT AXIALLY SYMMETRIC STATIONARY SOLUTIONS OF EINSTEIN'S EQUATIONS}

Some aspects of certain generation methods of new empty space solutions of Einstein's equations are analyzed. The methods are characterized in a generalized form by the forthe variables in the function $H$ are separable. The first theorem states that the algorithm in this particular case is equivalent to that of constructing a "generating potential» $\gamma$ in $\left[{ }^{2,5}\right], \gamma \neq$ const. From the second theorem, a number of useful formulae have been derived. If the initial solution is static, some specific formulae have been obtained for the calculation of the "generating potential» $x \equiv \alpha$ of the method elaborated in [5]. The operation $l$ (if $\gamma \neq$ const) and $S$, considered in $\left[{ }^{2}\right]$ as constituents of Bäcklund transformations, are studied in the case of the initial static metric as well as the flat one. The explicit representation of the constituents of the operation leading from the flat metric to that of Kerr, reveals the essence of the procedure. 